\title{
Problemática y tratamiento actual de la seudoartrosis de escafoides
}

\author{
A. Luis Calero ${ }^{(1)}$, A. Jiménez Guardeño ${ }^{(2)}$, G. Fernández Moreno ${ }^{(1)}$, \\ J. J. Quintana Cruz ${ }^{(1)}$
}

HOSPITAL INTANTA ELENA. HUELVA

(1) MIR SERVICIO COT

(2) FEA TRAUMATOLOGÍA

Correspondencia:

Dra. Antonia Luis Calero

Avda. Costa de la Luz, 21, $6^{\circ} \mathrm{D}$

21002 Huelva

Tel.: 658882482

e-mail: luiscaan@hotmail.com

La fractura de escafoides es la más frecuente del carpo y es inherentemente inestable, por lo que aún con tratamiento precoz puede evolucionar hacia la seudoartrosis hasta en un $55 \%$ de los casos.

El objetivo de este estudio es el análisis de las diferentes técnicas quirúrgicas empleadas para el tratamiento de las seudoartrosis de escafoides y de sus resultados. Para ello realizamos una búsqueda en la literatura científica actual y analizamos nuestros resultados durante un período de 4 años.

La elección de la técnica utilizada dependerá del tipo de fractura, localización y estado vascular del polo proximal.

Se ha utilizado la técnica de Matti-Russe, la técnica de Fisk Fernández, el injerto vascularizado, y la cruentación y osteosíntesis con tornillo. El abordaje fue volar en un $86,6 \%$ de los casos, utilizando injerto de cresta en un $66,6 \%$. El plazo de inmovilización postoperatoria fue de 12 semanas de media (rango 8-15), obteniéndose la consolidación en el $93 \%$ de los casos, con un nivel de satisfacción alto en el $13,3 \%$ de los pacientes y moderado en el $80 \%$. Únicamente un caso $(6,6 \%)$ no consolidó por fracaso de una cruentación e injerto de cresta ilíaca y osteosíntesis con tornillo de compresión tipo Herbert.

Palabras Clave: seudoartrosis de escafoides, injerto vascularizado, fracturas polo proximal.
Fractures of the scaphoid are the most frequent in the carpus and they are intrinsically unstable, therefore they can develop into a non union in up to $55 \%$ of cases in spite of early treatment.

The objective of this study is to analyse the different surgical techniques used for the treatment of scaphoid non-unions and their results. A literature search of recent scientific papers was carried out and the results for a period of 4 years are analyzed.

The choice of technique depends on the type of fracture, localization and vascular state of the proximal pole. Techniques used are Matti-Russe, FiskFernandez, vascularized bone grafts and debridment and osteosynthesis with screw. The approach was palmar in 86.6 of cases using graft from the iliac crest in $66.6 \%$. Average time of postoperative immobilization was 12 weeks (range 8-15), obtaining consolidation in $93 \%$ of cases, with a high level of patient satisfaction in $13.3 \%$ of cases and moderate level of satisfaction in $80 \%$. Only one case $(6.6 \%)$ did not unite for failure of debridement and iliac crest bone graft and fixation with a Herbert screw.

Key words: scaphoid non-union, vascularized bone graft, fractures of the proximal pole.

Rev. Iberam. Cir. Mano - Vol. 35 - Núm. 2 - Noviembre 2007 (59-66) 


\section{INTRODUCCIÓN}

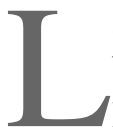

a seudoartrosis de escafoides es un problema frecuente y difícil de prevenir, por lo que nos planteamos distintas preguntas: ¿por qué fracasa un tratamiento inicial correcto y evoluciona hacia la seudoartrosis?, ¿qué técnica realizamos para el tratamiento de la seudoartrosis establecida?, y si también falla, ¿qué opciones de rescate se pueden aplicar?

Para su diagnóstico, además del antecedente de un traumatismo previo, podemos encontrar dolor localizado en el tubérculo del escafoides o dolor provocado por la compresión axial del primer metacarpiano. A la exploración puede existir desviación dorsal, deformidad en la tabaquera anatómica en un 22-68\% de los ca$\operatorname{sos}^{1}$, dolor a la dorsiflexión y disminución del arco de extensión de la muñeca.

Como pruebas complementarias se ha utilizado básicamente la $\mathrm{Rx}$, con las proyecciones para el escafoides, cuya valoración está sujeta a la experiencia del observador. La tomografía axial computerizada (TAC) permite valorar la anatomía ósea, el trazo de la seudoartrosis, la existencia de geodas y la confirmación de la falta de consolidación ${ }^{2}$. La resonancia magnética (RM) valora la vascularización del polo proximal, aunque su uso es motivo de controversia ${ }^{3}$.

Los factores de riesgo potenciales para la seudoartrosis son: insuficiencia del tratamiento inicial, ya sea por falta de diagnóstico o por tratamiento inadecuado, inmovilización de corta duración, desplazamiento del foco de fractura, fractura con diástasis mayor de $1 \mathrm{~mm}$, inestabilidad, ya sea ósea, por la propia configuración anatómica del escafoides o por reabsorción en la cara volar del foco de fractura, o mixta, cuando además se asocia a una lesión ligamentosa, factores vasculares o topográficos. La fractura del polo proximal ocasiona, entre el 16 y el $42 \%$ de los casos, una necrosis avascular ${ }^{4}$.

La seudoartrosis de escafoides se puede clasificar, según propone Mir et $\mathrm{al}^{5}$, en estable, sin pérdida de sustancia de hueso, o inestable, con pérdida de sustancia ósea.

Para que consolide la seudoartrosis es necesaria una adecuada vascularización, moduladores de la formación ósea, un aporte óseo mediante injerto y una estabilidad, bien con tornillos, centrados y de suficiente longitud que dan una mayor estabilidad, bien con agujas de Kirschner ${ }^{6}$.

Los métodos de tratamiento indicados en este tipo de patología son: fijación interna ${ }^{6,7}$, injerto óseo ${ }^{8,9}$, injerto óseo vascularizado pediculado $^{10}$, injerto óseo vascularizado de cresta ilíaca libre ${ }^{11}$, estimulación ósea y recientemente el uso de los factores de crecimiento ${ }^{12,13}$.

$\mathrm{Si}$ las técnicas anteriores no han sido eficaces empieza a producirse un estado degenerativo en la articulación radio-carpiana ${ }^{14}$ dando lugar a artrosis en la muñeca, dolor y colapso, lo que se conoce como Scaphoid Nonunion Advanced Collapse (SNAC). El método de rescate dependerá del momento evolutivo de la seudoartrosis. Las opciones técnicas son: tratamiento de los síntomas, estiloidectomía radial más reconstrucción del escafoides ${ }^{15}$, artrodesis parcial del carpo, carpectomía proximal, denervación de muñeca o artrodesis de muñeca ${ }^{16}$.

\section{MATERIAL Y MÉTODO}

Se han revisado todas las seudoartrosis tratadas en el Hospital Infanta Elena entre 20022006, que se han agrupado siguiendo la clasificación propuesta por Mir et al. ${ }^{5}$ El tratamiento aplicado dependió de los siguientes parámetros: desplazamiento de la seudoartrosis en el momento del diagnóstico, inestabilidad del carpo, vascularización del polo proximal y ausencia, o presencia, de cambios degenerativos.

El 46,6\% de los pacientes eran trabajadores de fuerza, el 26,6\% realizaban trabajos manuales y el $26,6 \%$ eran estudiantes.

El plazo de demora medio desde el traumatismo hasta el tratamiento quirúrgico fue de 54 meses (rango 3-180), por falta de diagnóstico inicial. El procedimiento terapéutico inicial de la fractura de escafoides que desarrollaría la seudoartrosis fue: $60 \%$ ninguno, pasó desapercibida, y $40 \%$ inmovilización, $30 \%$ de los cuales con un tiempo inferior a 2 meses ${ }^{17}$, aunque existen fracturas del escafoides del tercio distal, y algunas del tercio medio, que pueden consolidar en menos de 2 meses.

Una vez diagnosticada la seudoartrosis se ha clasificado como: estable el $20 \%$, e inestable el $80 \%$ de los casos (Tabla I). 
Tabla I - CRITERIOS DE INESTABILIDAD DE UNA SEUDOARTROSIS DE ESCAFOIDES

\begin{tabular}{|c|}
\hline separación $>$ de $1 \mathrm{~mm}$ \\
angulación intraescafoidea $>25^{\circ}$ \\
ángulo escafo-lunar $>60^{\circ}$ \\
ángulo luno-capital $>20^{\circ}$
\end{tabular}

A los 12 meses de la intervención todos los pacientes se evaluaron físicamente, analizando el dolor y la demanda de analgésicos que precisaban, la funcionalidad de la mano y la consolidación de la fractura. El dolor fue valorado utilizando la escala visual analógica (EVA); se midió la fuerza con un dinamómetro Jamar; y se realizó un estudio radiográfico, considerando consolidación ósea el paso de trabéculas óseas por el foco de fractura.

A los dos años de la intervención fueron entrevistados telefónicamente, para preguntar acerca de la satisfacción postintervención.

\section{RESULTADOS}

En total se evaluaron 15 pacientes, todos varones, con una edad media de 25 años (rango 16-38); la mano dominante era en todos los casos la derecha; en el $56,25 \%$ de los casos estaba afectada la muñeca derecha y en el $43,75 \%$ la izquierda, 1 caso era bilateral.

El mecanismo de producción de la fractura fue: en el 53,3\% caída, 20\% accidentes de tráfico y $20 \%$ traumatismo directo.

El diagnóstico de la seudoartrosis fue radiológico en todos los casos, midiendo además los ángulos escafo-lunar, capito-lunar, e intraescafoideo, apoyado con TAC en el 26,6\% de los casos y por RM en el $20 \%$. La localización de la seudoartrosis fue fundamentalmente en el cuerpo, 53,3\% de los casos (Tabla II), con la dirección de la línea de fractura transversa en el 73,3\% de los casos. Durante la cirugía se apreció esclerosis del polo proximal $(46,6 \%)$, cambios degenerativos en la estiloides radial $(33,3 \%)$ y seudoartrosis desplazadas más de 1 $\mathrm{mm}(60 \%)$.

\section{Tabla II - LocALIZACIÓN ANATÓMICA DE LA SEUDOARTROSIS DE ESCAFOIDES EN RX AL DIAGNÓSTICO}

\begin{tabular}{|lr|}
\hline Polo proximal & $40 \%$ \\
\hline Cuerpo & $53,3 \%$ \\
\hline Polo distal & $6,6 \%$ \\
\hline
\end{tabular}

Los procedimientos quirúrgicos utilizados fueron: reducción a cielo abierto por vía volar, cruentación de los extremos de la seudoartrosis y colocación de injerto, según la técnica de Matti-Russe, en los casos que cumplían un solo criterio de inestabilidad: separación mayor de $1 \mathrm{~mm}$ (26,6\% de los casos); reducción abierta por vía volar, cruentación del foco de seudoartrosis, interposición de injerto entre los extremos y osteosíntesis, según la técnica de Fizt Fernández, en los casos con más de un criterio de inestabilidad (46,8\%); injerto vascularizado de la arteria dorsal supraretinacular de radio distal, en un solo caso, con déficit de aporte sanguíneo en polo distal objetivado durante la cirugía $(6,6 \%)$; y cruentación y osteosíntesis sin aporte de injerto en el $20 \%$ en los casos de seudoartrosis de escafoides estables. Añadiendo estiloidectomía radial en el 33\% de los casos, en los que se apreciaban, en los estudios radiográficos previos, signos artrósicos, como afilamiento de estiloides radial o esclerosis subcondral. El abordaje fue volar en el $86,6 \%$ de los casos. El injerto se tomó de cresta ilíaca en un 66,6\% de los casos, ya que de esta localización podemos obtener injertos de hueso esponjoso denso y de buena calidad con cortical gruesa, y de la metáfisis del radio distal en el $13,3 \%$. Se utilizó como método de fijación las agujas de Kirschner en un $80 \%$ de los casos, y tornillos de compresión, tipo Herbert, en el 20\% restante. El empleo de agujas requiere una menor complejidad técnica, y, al dejarlas subcutáneas, pueden retirarse posteriormente con anestesia local, y no necesitan la apertura de la articulación escafo-trapezial. Está demostrado que el uso de agujas en lugar de tornillos sólo influye en el tiempo de inmovilización postoperatoria, que es más prolongado en el caso de las agujas, pero 
Tabla III - CoMpaRAción DE LOS ÁNGULOS ESCAFO-LUNAR Y TRANSESCAFOIDEO PRE Y POSTOPERATORIOS

\begin{tabular}{|ccc|}
\hline Preoperatorio & Postoperatorio & \\
\hline $58^{\circ}\left(\right.$ rango $\left.32,5-80^{\circ}\right)$ & $45^{\circ}\left(\right.$ rango $\left.28-64^{\circ}\right)$ & ángulo escafo-lunar \\
\hline $54^{\circ}\left(\right.$ rango $\left.0-132^{\circ}\right)$ & $9^{\circ}\left(\right.$ rango $\left.0-25^{\circ}\right)$ & ángulo transescafoideo \\
\hline
\end{tabular}

no influye en el resultado, es decir, el porcentaje de consolidación de la fractura es el mismo con los dos tipos de osteosíntesis ${ }^{6}$.

Se realizó una inmovilización postoperatoria en todos los casos, durante un período medio de 3 meses, 2 semanas con férula antebraquial almohadillada con inmovilización del primer dedo, y las 10 semanas posteriores, como media (rango 6-12 semanas), con yeso antebraquial incluyendo F1 del primer dedo, hasta que con las pruebas radiográficas se objetive el paso de trabéculas óseas por el foco de fractura.

En los casos en los que se emplearon agujas de Kirschner, el tiempo medio de retirada de las agujas fue de 4 meses (rango 3-6), que es 4 semanas (rango 2-5) después de la retirada de la inmovilización, para mantener la estabilidad cuando se inicia la movilización activa.

Fue preciso derivar al servicio de Rehabilitación al 46,6\% de los pacientes intervenidos.

Sólo un caso, al que se practicó cruentación e injerto de cresta ilíaca y osteosíntesis con tornillo de compresión tipo Herber, no consolidó la seudoartrosis, proponiéndose una cirugía de rescate mediante artrodesis de las 4 esquinas, sin embargo el paciente rehusó operarse.

Además del estudio radiológico en pre y postoperatorio, en el 13,3\% de los casos se practicó una TAC, que es la mejor técnica para evaluar la consolidación, pues la exploración radiográfica puede dar un $25 \%$ de falsos negativos $^{2}$, y en el 6,6\% una RM con gadolinio, para determinar la viabilidad del polo distal del escafoides tras injerto vascularizado.

El seguimiento post intervención quirúrgica ha sido de 9,4 meses de media (rango 8-18).

Los resultados clínicos obtenidos fueron: molestias tolerables, que precisaban AINEs ocasionalmente, EVA 2-3 en el $89 \%$ de los casos,
4-5 el 11\% restante; un balance articular de: flexión dorsal $20^{\circ}$ (rango $0-30^{\circ}$ ), flexión volar $40^{\circ}$ (rango $10-60^{\circ}$ ), en el $95 \%$ de los pacientes la prono-supinación estaba libre y conservada, en el 5\% restante presentaban una supinación de $45^{\circ}$ (rango $10-60^{\circ}$ ), y una pronación de $30^{\circ}$ (rango $5-35^{\circ}$ ); y ligera pérdida de fuerza sin repercusión en las actividades de su vida diaria, el promedio de fuerza fue de $26 \mathrm{~kg}$ en la mano intervenida (rango 18-30) sobre $34 \mathrm{~kg}$ de la mano contralateral (rango 28-40). En el paciente intervenido de forma bilateral la fuerza era de $26 \mathrm{~kg}$ en la mano izquierda y $30 \mathrm{~kg}$. en la mano derecha, siendo la mano derecha la dominante.

El $65 \%$ de los pacientes volverían a operarse, el $35 \%$ restante no se volverían a operar por las molestias postoperatorias y la rehabilitación posterior, encontrando un nivel de satisfacción alto en el 13,3\% de los pacientes, moderado en el $80 \%$, por el período de inmovilización, e insatisfechos en el 6,6\% (1 caso).

En el 93,3\% de los pacientes volvieron a su actividad laboral previa a la cirugía, el paciente que no consolidó $(6,6 \%)$ refería dolor en la muñeca, con incapacidad para trabajar, por lo que se propuso cirugía de rescate que el paciente rechazó.

Se objetivó una mejora de los ángulos comparados con el preoperatorio (Tabla III), no se pudo valorar el ángulo capito-lunar, que es el que más se afecta, debido a la falta de proyecciones laterales estrictas (Figuras 1 y 2), tampoco se pudieron medir en la TAC o RM realizadas en algunos casos, ya que en un caso no consolidó y en el otro hubo un retraso de consolidación. En el 80\% de los casos el diagnóstico de consolidación fue radiológico, valorando la consolidación por el paso de trabéculas 


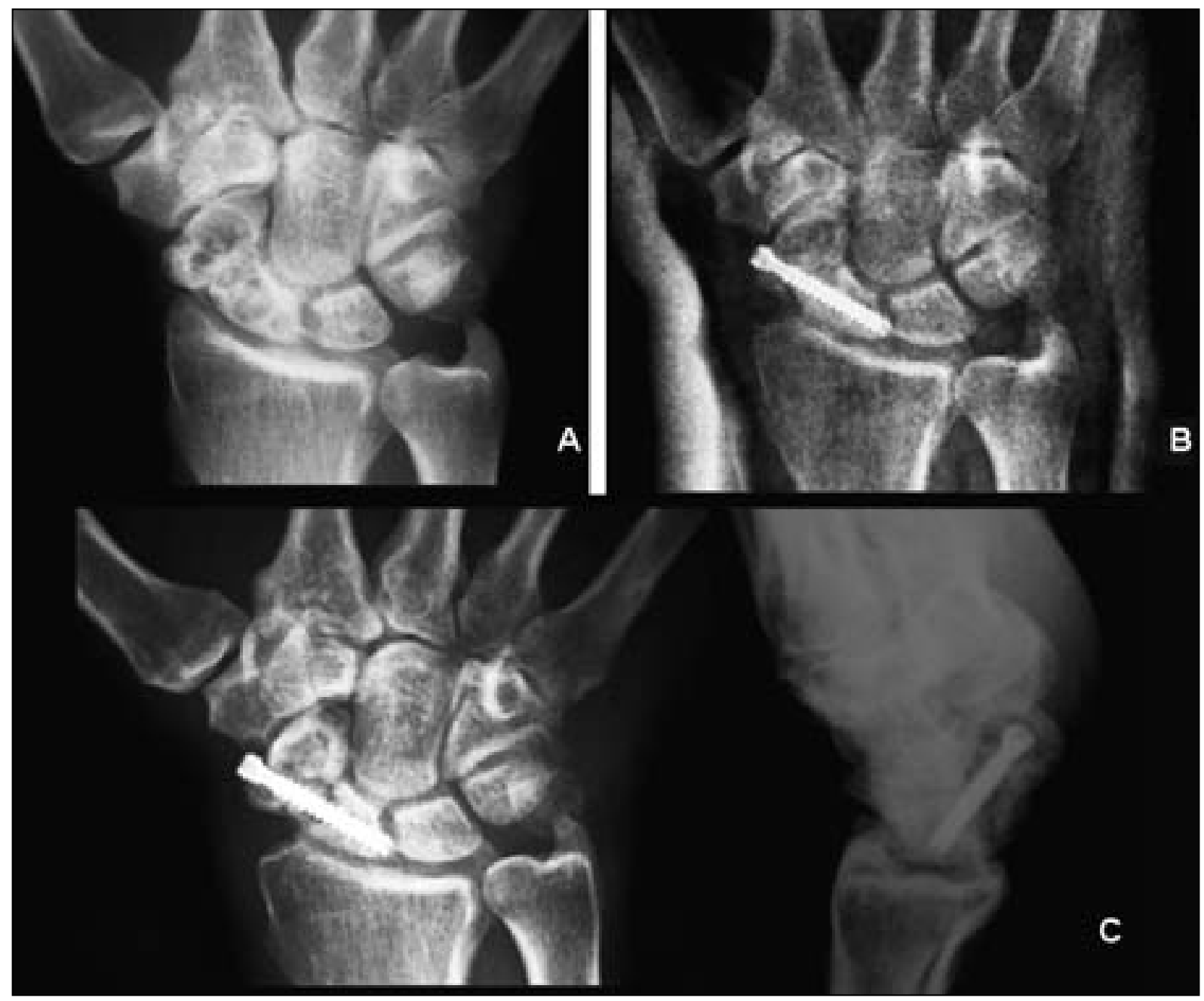

Figura 1: Varón de 38 años que consulta por dolor a nivel de tabaquera anatómica. Antecedente traumático hace meses sin tratamiento inicial. A: Rx AP inicial. B: reducción abierta y osteosíntesis con tornillo de compresión (BOLD ${ }^{R}$, Osteosan). C: Rx 7 meses después de la cirugía donde no se observa consolidación de la fractura.

óseas por el foco de fractura, en el $13,3 \%$ la consolidación se diagnosticó por TAC, ante imágenes no concluyentes en las radiografías en un plazo medio de 12 semanas (rango 6-16), y un caso $(6,6 \%)$ no consolidó.

\section{DISCUSIÓN}

Existen controversias en el tratamiento de la seudoartrosis de escafoides ${ }^{16}$. Es sintomático en cerca del $67 \%$ de los casos y cuando se hace necesaria una cirugía de rescate se consiguen muñecas asintomáticas en un alto porcentaje ${ }^{17}$
En los pacientes de la serie sólo en 1 caso $(6,6 \%)$ se obtuvo un mal resultado, después de la cirugía de la seudoartrosis, por no consolidación, porcentaje que coincide con la bibliografía consultada ${ }^{4-10,16,18-21}$.

Escoger el procedimiento más adecuado es imperativo para obtener buenos resultados, los parámetros más importantes a tener en cuenta son: valorar si el escafoides es estable o inestable. La técnica de Fitz Fernández con injerto de interposición está indicada en los casos en los que exista inestabilidad, angulación dorsal de la unión semilunar-polo proximal del escafoides; la técnica de Matti-Russe si es mínima- 


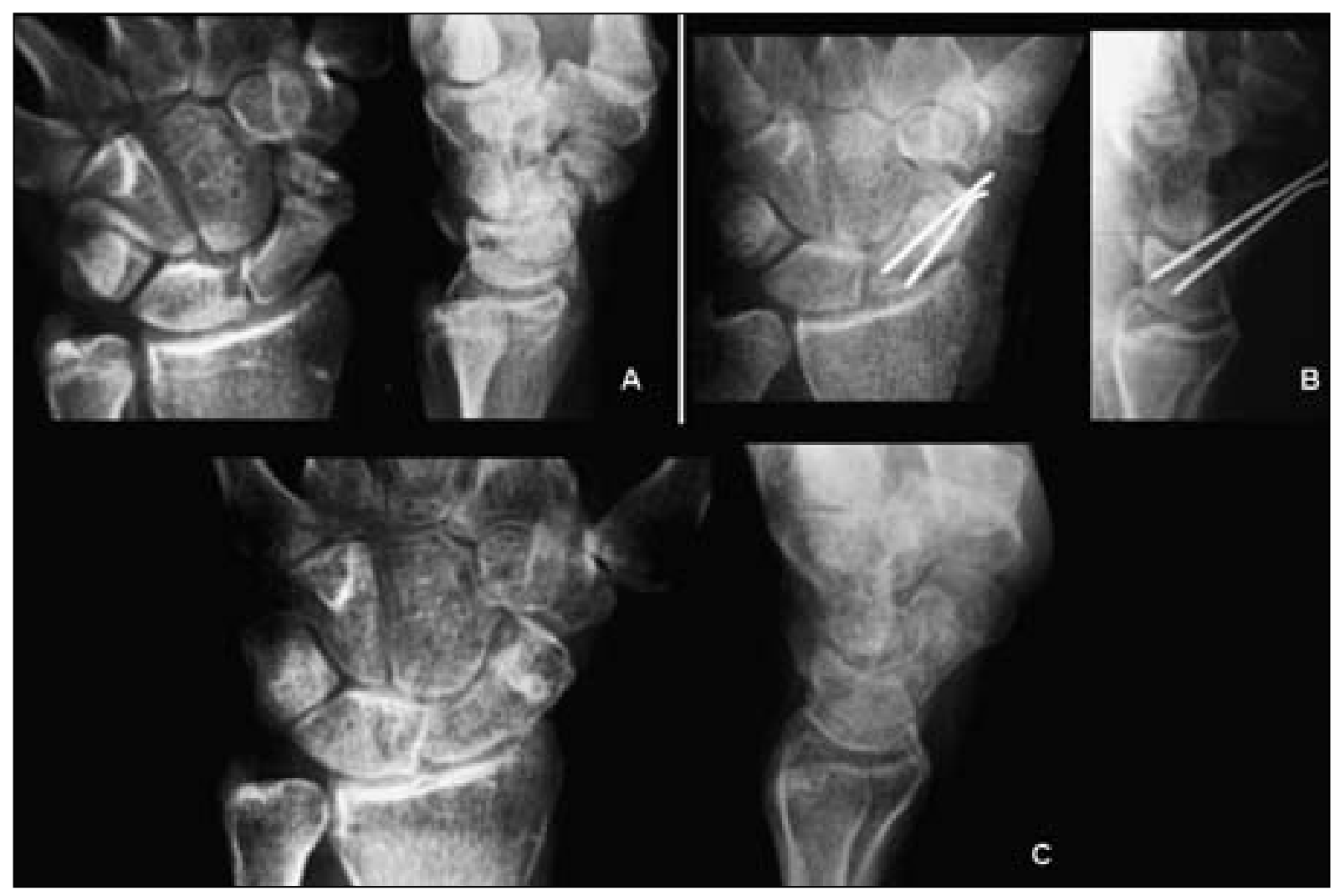

Figura 2: Varón de 17 años que acude por dolor en tabaquera anatómica de la mano izda. Refiere una caída de motocicleta tras la cual fue inmovilizado por un tiempo menor de 3 semanas. A: Rx anteroposterior y lateral inicial. B: control postquirúrgico inmediato tras cruentación de foco y osteosíntesis con 2 agujas de Kirschner. C: Rx 5 meses postintervención, en donde se observa consolidación de la seudoartrosis.

mente inestable, el escafoides mantiene su eje y su forma normal, y el ángulo escafo-lunar es normal; o la osteosíntesis con tornillo a compresión ${ }^{19}$ si es estable; si hay resorción ósea, cambios quísticos o pequeña deformidad angular, se podría realizar injerto en cruz maltesa ${ }^{18}$; si hay ausencia de una buena vascularización en el polo proximal ${ }^{20}$, o por fracaso de injertos convencionales, se puede realizar un injerto vascularizado $^{21}$, aunque existen autores que indican este tipo de injertos como tratamiento inicial $^{22}$.

En los últimos diez años se han propuesto numerosos métodos de injerto óseo vascularizado obtenido de la epífisis radial con la rama radial carpal volar ${ }^{23}$; del pronador cuadrado con su pedículo más un fragmento óseo de la estiloides radial volar ${ }^{24}$; cúbito distal vascularizado por la arteria cubital ${ }^{25}$, y de la rama ascendente de la arteria radial con injerto radial ${ }^{26}$.
Si encontramos cambios degenerativos en la estiloides radial, además de la reconstrucción del escafoides se realizará una estiloidectomía asociada $^{1}$. Si existe artrosis capito-lunar, lo más adecuado sería emplear procedimientos de rescate $^{1}$, como son la artrodesis capito-lunar con resección del escafoides, o la artrodesis en cuatro esquinas. Si existe un SNAC se realizaría carpectomía proximal, artrodesis parcial, artrodesis capito-lunar, si no hay afectación de la articulación radio-lunar, más resección del escafoides. La resección del escafoides puede ser completa o parcial dejando la parte distal del escafoides. También puede emplearse la denervación de muñeca, como gesto asociado a las técnicas anteriores o como tratamiento aislado, buscando aumentar el bienestar del paciente, sin tratar en manera alguna la causa generadora de los dolores, usándose en los casos en los que no es posible realizar ninguna de las interven- 
ciones de reconstrucción; y en casos muy avanzados de artrosis carpiana, la artrodesis de muñeca.

En los últimos tiempos se han desarrollado nuevas técnicas, algunas coadyuvantes, como es el uso de ultrasonidos de baja intensidad ${ }^{27}$ y el desarrollo de implantes biodegradables, como el ácido poliláctico reforzado, con el que algunos autores consiguen tasas de consolidación en todos los $\operatorname{casos}^{28}$, pero otros autores refieren que el tiempo de reabsorción de estos implantes es insuficiente para asegurar la consolidación ósea ${ }^{29}$, también es preciso señalar el uso de proteína morfogenética humana $^{12,13}$.

\section{CONCLUSIÓN}

Como conclusión, el tratamiento de la seudoartrosis de escafoides continúa siendo un desafío. Resaltar la gran importancia de conocer la anatomía y biomecánica para una correcta comprensión de la fisiopatología; la importancia de un diagnóstico y tratamiento precoz para evitar la progresión de los cambios degenerativos; una planificación preoperatoria adecuada, valorando el abordaje necesario, el empleo de injerto, de material de osteosíntesis; el uso de coadyuvantes como los ultrasonidos o la proteína morfogenética humana y tener siempre presente la posibilidad de un tratamiento reconstructivo o de rescate.

\section{BIBLIOGRAFÍA}

1. Merle M, Dautel G, Loda G. Complicaciones de las fracturas del escafoides. Mano traumática. Cirugía secundaria muñeca traumática. Masson. Ed Panamericana: Barcelona. 1996; 16: 341-62.

2. Soler JM, González J, Pérez R et al. Computed Tomography in partial Carpal arthrodesis. J Hand Surg B, 1997; 22: 653-5.

3. Singh AK, Davis TCR, Dawson JS, et al. Gadolinium enhanced MR assessment of proximal fragment vascularity in nonunions after scaphoid fracture: does it predict the outcome of reconstrive surgery?. J Hand Surg B, 2004; 29: 444-8.

4. Muzaffar A, Carter P. Vascularized bone grafting and Herbert screw fixation of scaphoid nonunions with avascular proximal poles. Tech Hand Upper Extrem, 2002; 6: 155-64.

5. Mir J, Font J, Aizpurna J, et al. Clasificación de las fracturas y pseudoartrosis del escafoides carpiano. Rev Ortop Traumatol, 1998; 42: 8-16.

6. Zichner L, Rehar, S. Treatment of scaphoid non-unions. J Bone Joint Surg B, 2001; 83 Suppl II: 222.

7. Trumble $\mathrm{T}$, Clarke $\mathrm{T}$, Kreder $\mathrm{H}$. Non-union of the scaphoid.
Treatment with cannulated screws compared with treatment with Herbert screws. J Bone Joint Surg A, 1996; 78: 1829-37.

8. Le Bellec Y, Touan C, Alnot JY, et al. Treatment of nonunions of the scaphoid without necrosis or osteoarthritis using a corticocancellous graft: a series of 40 cases. J Bone Joint Surg B, 2001; 83 Suppl I: 23.

9. Daly K, Gill P, Magnussen PA, et al. Established nonunion of the scaphoid treated by volar wedge grafting and Herbert screw fixation. J Bone Joint Surg B, 1996; 78: 530-4.

10. Chen A, Chao E, Tu Y, et al. Scaphoid nonunion treated with vascular bone grafts pedicled on the dorsal supra-retinacular artery of the distal radius. J Trauma, 2006; 61: 1192-7.

11. Harpf C, Gabl M, Reinhart C,.et al. Small Free Vascularized iliac crest bone grafts in reconstruction of the scaphoid bone: A retrospective study in 60 cases. Plast Reconstr Surg, 2001: 108: 664-74.

12. Jones NF, Brown EE, Mostofi $A$, et al. Healing of a scaphoid nonunion using human bone morphogenetic protein. $J$
Hand Surgery A, 2005; 30: 528-33.

13. Bilic $R$, Simic $P$, Jelic $M$, et al. Osteogenic protein I (BMP-7) accelerates healing of scaphoid nonunion with proximal pole sclerosis. Int Orthop, 2006; 30: 128-34.

14. Moritomo $\mathrm{H}$, Tada $\mathrm{K}$, Yoshida $\mathrm{T}$, et al. The relationship between the site of nonunion of the scaphoid and scaphoid nonunion advanced collapse (SNAC). J Bone Joint Surg B, 1999; 81: 871-6.

15. Malerich, M, Littler J, Eaton R. Distal scaphoid resection arthroplasty for the treatment of patients with degenerative arthritis secondary to scaphoid nonunion. Tech Hand Upper Extrem, 2002; 6: 98-102.

16. Smith $S$, Cooney $P$, Revision of failed bone grafting for nonunion of the scaphoid: Treatment options and results. Clin Orthop, 1996; 327: 98-109.

17. Carrozzella J, Stern PJ. Treatment of comminuted distal radius fractures with pins and plaster. Hand Clin, 1988; 4: 391-7.

18. Walsh C, Hunt T. Management of acute and chronic scaphoid fractures. Curr Opinion Orthop, 1999; 10: 271-6. 
19. Rajagopalan B, Squire D, Samuels LO. Results of Herbertscrew fixation with bone-grafting for the treatment of nonunion of the scaphoid. $J$ Bone Joint Surg A, 1999; 81: 48-52.

20. Kirkeby R. Vascularised bone graft for the treatment of nonunion of the scaphoid. Scand $J$ Plast Reconstr Surg Hand Surg, 2006; 40: 240-3.

21. Chang MA. The outcomes and complications of 1,2-intercompartmental supraretinacular artery pedicled vascularized bone grafting of scaphoid nonunions. J Hand Surg A, 2006; 31: 387-96.

22. Aguilella L, Fargueta I, Blasco, $\mathrm{C}$, et al. Injertos óseos vascu- larizados en el tratamiento de la pseudoartrosis de escafoides. Rev Ortop Traumatol, 2002, 4: 311-6.

23. Kuhlmann JN, Mimoun M, Boabighi $A$, et al. Vascularized bone graft pedicled on the volar carpal artery for nonunion of the scaphoid. J Hand Surg B, 1987; 12: 203-10.

24. Kawai H, Yamamoto K. Pronator quadratus pedicled bone graft for old scaphoid fracture. J Bone Joint Surg B, 1988; 70: 829-31.

25. Guimberteau JC, Panconi B. Recalcitrant non-union of the scaphoid treated with a vascularized bone graft based on the ulnar artery. J Bone Joint Surg A, 1990; 72: 88-97.
26. Zaidemberg C, Siebert JW, Angrigiani $C$. A new vascularized bone graft for scaphoid nonunion. J Hand Surg A, 1991; 16: 474-8.

27. Monrea, R. The effect of ultrasound on the healing of muscle-pediculate bone graft in scaphoid nonunion. Int Orthop, 2006; 30: 123-7.

28. Akmaz I, Kiral A, Pehlivan O, et al. Biodegradable implants in the treatment of scaphoid nonunion International. Orthopaedics, 2004; 28: 261-6.

29. Yañez AJ, Castro M, Pombo $S$, et al. Osteosíntesis con tornillo del polo proximal del escafoides carpiano. Rev Ortop Traumatol, 2005; 49: 281-6. 